\title{
ESTILOS DE VINCULAÇÃO, ORIENTAÇÃO PARA O TRABALHO E RELAÇÕES PROFISSIONAIS
}

\author{
Marisa Fonseca ${ }^{1}$ \\ Isabel Soares ${ }^{2}$ \\ Carla Martins $^{3}$
}

Resumo: O presente estudo procura analisar as relações entre os estilos de vinculação e a qualidade das relações profissionais e com o trabalho. A maioria dos participantes do estudo - quinhentos e noventa e oito indivíduos - identifica-se com um estilo de vinculação segura e com uma orientação para o trabalho segura, tendo sido encontrada uma associação significativa entre estas duas variáveis. Verificou-se, ainda, que os indivíduos com um estilo seguro, quer ao nível da vinculação, quer ao nível da orientação para o trabalho, manifestam maior satisfação profissional e melhor adaptação ao trabalho.

Palavras-chave: estilos de vinculação, orientação para o trabalho

Attachment styles, work orientation and relations (Abstract): The present study was aimed to examine the relations between attachment styles and the quality of work relations. Most of the participants - five hundred and ninety-eight adults - show a secure attachment style and a secure work orientation. A positive association was found between these two variables. Participants with a secure attachment style and work orientation show higher levels of satisfaction and adaptation related to their work.

Key-words: attachment styles, work orientation

\footnotetext{
${ }^{1}$ Psicóloga, doutoranda da Facultat de Psicologia, Ciències de l'Educació i de l'Esport Blanquerna, Universitat Ramon Llull. A informação sobre o artigo poderá ser solicitada pelo e-mail marisaegipto@hotmail.com.

2 Professora Catedrática do Departamento de Psicologia, Instituto de Educação e Psicologia, Universidade do Minho.

${ }^{3}$ Professora Auxiliar do Departamento de Psicologia, Instituto de Educação e Psicologia, Universidade do Minho.
}

PSICOLOGIA, Vol. XX (1), 2006, Edições Colibri, Lisboa, pp. 187-208 


\title{
Introdução
}

Ao longo dos tempos, a Teoria da Vinculação vem sendo reconhecida na comunidade científica como uma perspectiva desenvolvimental de imprescindível valor para a compreensão do ciclo de vida:

\begin{abstract}
Attachment theory is among the most sweeping, comprehensive theories in psychology today. It offers a biosocial, lifespan account of how close relationship form, and maintained, and dissolve and how relationship influence, sometimes permanently, the persons involved in them. The theory addresses these issues from a variety of perspectives, including physiological, emotional, cognitive and behavioural. The theory articulates constructs and processes that are relevant to understanding elements of social development, interpersonal behaviour, relationship functioning, psychosocial adjustment and clinical disorders. (Rholes \& Simpson, 2004, p. 3).
\end{abstract}

Ao considerarmos a vinculação numa perspectiva de desenvolvimento do ciclo vital, os diferentes contextos de vinculação (relações familiares, amorosas ou de amizade íntima) podem ser palco de estabilidade e manutenção dos modelos internos dinâmicos de vinculação construídos previamente ou uma oportunidade de mudança e de transformação pela integração de novos elementos, de modo a actualizar e adaptar esses modelos às novas exigências internas ou externas. $\mathrm{O}$ processo desenvolvimental integra, simultaneamente, continuidade e mudança (Sroufe, Egeland, Carlson \& Collins, 2005), nada se mantém igual ou inalterável, uma vez que diferentes idades exigem diferentes estratégias, mas nada se exclui pois há transformação e reconstrução. Os modelos internos dinâmicos existem no presente, estão entrelaçados no passado e direccionados para o futuro. São dimensões centrais da personalidade que moldam a forma como o sistema de vinculação se manifesta, ao direccionar os padrões de respostas cognitivas, emocionais e comportamentais relacionadas com a vinculação nos vários contextos de vida.

\section{Vinculação: da infância à idade adulta}

No percurso de vida do ser humano, o desenvolvimento de relações de vinculação ocorre inicialmente entre crianças e seus progenitores e, mais tarde, também entre adultos (Bowlby, 1973). Ao longo do desenvolvimento, a complementaridade de papéis vai dando lugar à reciprocidade, $\mathrm{o}$ nível representacional vai ganhando mais relevo e articulação com a manifestação dos comportamentos de vinculação e a necessidade de presença física, para obtenção de conforto e alívio em situações adversas, vai podendo ser substituída, pelo menos em algumas situações, pela presença simbólica ou pela interacção à distância. 
O sistema comportamental de vinculação funciona em estreita ligação com o sistema comportamental de exploração, estando a sua activação subordinada à sensação de segurança, disponibilidade e responsividade da figura de vinculação. Em particular na infância, em situações de stress, a criança tem que se sentir segura para que seja desactivado o sistema comportamental de vinculação e activado o sistema de exploração. Na infância, de modo bem diferente da idade adulta, nas situações de stress, o sistema de vinculação é soberano face aos outros sistemas, estando este peso relativo relacionado etiologicamente com a necessidade de sobrevivência da espécie.

$\mathrm{Na}$ idade adulta, o sistema comportamental sexual tem também um papel de relevo, sendo que nas relações duradouras tende a fortalecer a ligação emocional entre os parceiros e a promover o desenvolvimento de uma relação de vinculação.

Com o crescimento, o ser humano cria novas relações de vinculação, essencialmente no âmbito de relações de intimidade, podendo estas ser de amizade ou de amor romântico. Algumas relações de amizade, desenvolvidas pelo jovem adulto e pelo adulto, podem constituir-se como verdadeiras relações de vinculação, na medida em que há procura de proximidade, prestação de cuidados, protecção, sentimento de segurança, ansiedade perante a ameaça de separação e dor na possibilidade de perda ou de separação. Neste sentido, Ainsworth (1989) refere haver evidência para acreditar que algumas (não todas) relações de amizade constituem laços afectivos duradouros. A vinculação a uma nova figura não implica que a vinculação aos pais tenha desaparecido (Ainsworth, 1989), pois a maioria dos adultos continua com uma relação significativa de vinculação com os pais. Além disso, em determinada altura da idade adulta a relação de vinculação entre os filhos e os pais tende a evoluir no sentido da simetria ao nível da manifestação dos sistemas de vinculação e de prestação de cuidados e, num período mais tardio do ciclo vital, com os pais, numa fase de maior dependência, poderá desenvolver-se no sentido de uma relação assimétrica, mas agora no sentido inverso à que ocorreu na infância.

Bowlby (1969/82) refere a existência de relações de vinculação da criança não apenas com uma figura de vinculação, mas com um número restrito de adultos, sendo construída uma hierarquia de preferências, que se manifesta, de uma forma mais clara, em situações de adversidade, quando a criança procura a proximidade com a figura que ocupa o primeiro lugar da hierarquia e, não a encontrando, recorre a uma segunda figura, e assim sucessivamente. Existe o mesmo pressuposto para a idade adulta, a ligação a uma nova figura de vinculação não faz desaparecer as anteriores, mas implica uma reorganização hierárquica das figuras de vinculação. Pode mesmo ocorrer que esta hierarquização seja distinta consoante a natureza da situação adversa sentida pelo adulto. 


\section{Estilos de vinculação no adulto}

Cindy Hazan e Philipp Shaver (1987) foram pioneiros na exploração dos estilos de vinculação no adulto, com base nos pressupostos da Teoria da Vinculação de Bowlby $(1969,1979)$, que descreve o relacionamento amoroso no adulto como um processo de vinculação, e de Ainsworth (1989), e reiteram a afirmação, alargando a sua conceptualização às relações íntimas de amizade:

Affectional bonds and subjectives states of strong emotions tend to be together, but every novelist and playwright Knows. Thus, many of the most intense of all human emotions arise during the formation, the maintenance, the disruption and the renewal of affectional bonds - Which for that reason, are sometimes called emotional bonds. In terms of subjective experience, the formation of a bond is described as falling in love, maintaining a bond as loving someone and losing a partner as grieving over someone. Similarly, the threat of loss arise anxiety and actual loss causes sorrow; whilst both situations are likely to arouse anger. Finally, the unchallenged maintenance of a bond is experienced as a source of security, and the renewal of a bond as a source of joy. (Bowlby, 1979, p. 69).

Nos estudos das relações íntimas enquanto processo de vinculação, Hazan e Shaver (1987), Shaver e Hazan (1988) e Shaver, Hazan e Bradshaw (1988) identificaram a versão adulta de três estilos de vinculação (seguro, inseguro-ambivalente e inseguro-evitante), traduzindo as descrições de Ainsworth, Blehar, Waters e Wall (1978) das crianças na Situação Estranha, em termos desenvolvimentalmente adaptados para as relações entre adultos (cf. Quadro 1).

De acordo com investigações de Hazan e Shaver (1987), Shaver e Hazan (1988) e Shaver, Hazan e Bradshaw (1988), os indivíduos classificados com um estilo de vinculação segura são caracterizados por estabelecerem facilmente relações de proximidade com outras pessoas e por se sentirem confortáveis com a intimidade que lhes é inerente. As suas relações, em termos de sistema de prestação de cuidados, são de reciprocidade, respondendo à adversidade sentida (por si ou pelo outro), cuidando ou permitindo ser cuidados numa alternância de papéis que se adequa ao contexto relacional. Por se considerarem pessoas passíveis de serem amadas, não se mostram preocupados com a possibilidade de serem abandonados pelas suas figuras de vinculação. As figuras de vinculação constituem para eles uma base segura nas situações de adversidade, sendo percepcionadas como sensíveis e responsivas face às necessidades experenciadas.

Os indivíduos com um estilo de vinculação inseguro-ansioso/ambivalente manifestam dificuldade na gestão da proximidade com as figuras 
de vinculação; conscientes da sua própria necessidade de uma maior proximidade, receiam que este aspecto leve ao afastamento e perda da amizade/amor das figuras significativas; frequentemente estabelecem relações assimétricas em termos de cuidados, cuidando ou sendo cuidados de forma quase compulsiva, com uma reduzida flexibilidade cognitiva/emotiva para a alternância de papéis.

Quadro 1: Estilos de vinculação segundo Hazan e Shaver (1987), Shaver e Hazan (1988) e Shaver, Hazan e Bradshaw (1988)

Considero ser relativamente fácil ficar próximo(a) de outras pessoas e sinto-me confortável quando dependo delas. Habitualmente não me preocupo com a possibilidade de ser abandonado(a) ou de alguém se aproximar demasiado de mim.

Acho que as outras pessoas estão relutantes em ficar tão próximas de mim como eu gostaria. Preocupo-me, muitas vezes, que o meu companheiro (minha companheira) não me ame realmente, que não me queira, ou que não queira ficar comigo. Quero ficar muito próximo(a) do meu companheiro(minha companheira) e este desejo, às vezes, afasta-o(a).

Inseguro-evitante

Sinto-me algo desconfortável ao ser próximo(a) de outras pessoas. Sinto dificuldade em confiar nelas completamente e a permitir a mim próprio depender delas. Fico nervoso(a) quando alguém fica demasiado próximo de mim e, muitas vezes, os meus companheiros amorosos querem que eu seja mais intimo(a) do que me sinto confortável a ser.

O estilo de vinculação evitante é caracterizado pelo desconforto que os indivíduos percepcionam em relação à proximidade e à intimidade que as relações com as figuras significativas podem implicar. Estes indivíduos tendem a evidenciar dificuldade em confiar no outro, uma vez que percepcionam as figuras de vinculação como não responsivas em situações de adversidade; cuidar e ser cuidado é algo que tendem a evitar, uma vez que percepcionam este factor como uma dependência desagradável.

A pesquisa de Hazan e Shaver apresenta uma distribuição dos três estilos de vinculação (evitante, seguro, ansioso/ambivalente) similar às referidas em estudos com amostras normativas de crianças americanas 
(Campos, Barrett, Lamb, Goldsmith \& Stenberg, 1983), por outro lado, resultados similares foram encontrados em outros estudos com adultos (Feeney \& Noller, 1990; Levy \& Davis, 1988; Mikulincer, Florian \& Tolmacz, 1990).

\section{Exploração na criança e no adulto}

Tal como foi referenciado, o sistema comportamental de exploração funciona de modo complementar ao sistema de vinculação: se a activação do sistema de vinculação aumenta de intensidade, diminui a activação do sistema exploratório e, desta forma, há uma menor atenção aos outros, aos aspectos do meio ambiente, o indivíduo explora menos e aprende menos (Ainsworth \& Bell, 1970). Se a interligação dos sistemas ocorrer neste sentido com muita frequência, diminui a competência do indivíduo porque restringe as oportunidades de aprendizagem (ao nível de conteúdos, mas também ao nível das relações e da socialização). Tal como refere Jongenelen (2004), a exploração de novos estímulos tende a ocorrer quando a acessibilidade da figura de vinculação está assegurada, o sistema de prestação de cuidados está medianamente activado, o sistema de vinculação está desactivado e o sistema exploratório activado. Desta forma, pode conceber-se o sistema de vinculação como possibilitando a manutenção da proximidade e a obtenção de segurança que permite a exploração.

De acordo Hazan e Shaver (1990), na actividade profissional, os indivíduos com estilos de vinculação seguros têm auto-relatos de sucesso e de satisfação laboral, menor medo de falhar e menor preocupação com o desempenho e com a avaliação dos colegas, apreciam as recompensas e a actividade exploratória per si.

De modo análogo ao evitamento manifestado em algumas crianças, cuja exploração parece ter como função evitar a proximidade e/ou contacto com a(s) figura(s) cuidadora(s), os adultos com estilo de vinculação evitante tendem a demonstrar uma atitude semelhante nas suas actividades de exploração, adoptam uma postura no trabalho de ocupação compulsiva que tem como função a distracção das necessidades individuais de vinculação e o evitar interacções desconfortáveis com os outros. Do ponto de vista da exploração, o estilo de vinculação evitante poderá estar associado a uma preferência em trabalhar sozinho e a trabalhar durante as férias como um pretexto para evitar socializar/conviver, com prejuízo da saúde e das relações íntimas e sociais (Hazan \& Shaver, 1990).

Os adultos com estilo de vinculação ansioso/ambivalente tendem a centrar-se na manutenção da proximidade com os seus cuidadores, para explorarem de forma eficaz. A exploração é utilizada como uma forma de realização para atrair a atenção e a aprovação da figura de vinculação, 
constituindo uma oportunidade de satisfazer as necessidades de vinculação até então não satisfeitas. A preocupação excessiva com as questões relacionadas com a vinculação constitui um distractivo da actividade profissional, estando associada a uma certa incapacidade de terminar projectos de trabalho, dificuldade em cumprir os prazos e a um fraco desempenho profissional. Nesta linha de pensamento, será compreensível que o estilo de vinculação ansioso/ambivalente esteja associado a uma preferência para trabalhar com outros mais do que sozinho e a ficar sobrecarregado com tarefas como forma de agradar aos outros (Hazan \& Shaver, 1990).

$\mathrm{Na}$ linha das investigações desenvolvidas por Hazan e Shaver (1990), o presente estudo pretende examinar as relações entre o estilo de vinculação em adultos e a sua orientação face ao trabalho.

Quadro 2: Características socio-demográficas da amostra

\begin{tabular}{lc}
\hline & Frequência \\
\hline Género & \\
\hline Masculino & $281(47,0)$ \\
Feminino & $317(53,0)$ \\
\hline Habilitações Académicas & \\
\hline Até ao 9. ${ }^{\circ}$ ano de Escolaridade & $144(24,8)$ \\
9. ${ }^{\circ}$ 12. ${ }^{\circ}$ Ano de Escolaridade & $149(25,6)$ \\
No decurso de Licenciatura & $64(11,0)$ \\
Licenciatura & $202(34,8)$ \\
Mestrado/Doutoramento & $22(3,8)$ \\
\hline Situação Profissional & $25(4,2)$ \\
\hline Estudante & $525(87,8)$ \\
No activo & $44(7,4)$ \\
Desempregado & $2(0,3)$ \\
Reformado & $262(43,8)$ \\
\hline Estado Civil & $308(51,5)$ \\
\hline Solteiro & \\
Casado/União de Facto & \\
Divorciado/Separado & \\
\hline & \\
\hline & \\
\hline
\end{tabular}




\section{Método}

\section{Participantes}

A amostra é constituída por 598 sujeitos, 53,0\% dos quais do sexo feminino. A idade dos participantes está compreendida entre os 18 e os 67 anos, com uma média de 34,5 anos $(D P=9,83)$. Relativamente às habilitações académicas, enquanto $24,8 \%$ frequentou ou completou a escolaridade mínima obrigatória $\left(9 .^{\circ}\right.$ ano), $34,8 \%$ concluiu a licenciatura e $3,8 \%$ tem mestrado ou doutoramento. Em termos profissionais, a vasta maioria da amostra pertence à população activa, havendo, na amostra, alguns casos de estudantes, desempregados e reformados. No respeitante ao estado civil, $43,8 \%$ dos indivíduos são solteiros, $51,5 \%$ casados ou vivem em união de facto e os restantes são divorciados, separados ou viúvos (Quadro 2).

\section{Medida}

Nesta investigação foi utilizada a versão portuguesa (Fonseca \& Soares, 2005) do Loving/Working: Are They Related?, de Hazan e Shaver (1990). Este instrumento de auto-relato pretende avaliar o estilo de vinculação e as temáticas relacionadas com relações amorosas/amor, bem como as relações profissionais, o trabalho e o lazer. No presente estudo foram utilizadas apenas a medida para avaliação do estilo de vinculação e as medidas relacionadas com a actividade profissional, as quais descrevemos em seguida:

\section{Avaliação da vinculação}

Medida Categorial de Avaliação do Estilo de Vinculação Adulta, na qual é solicitado ao indivíduo uma escolha forçada entre três parágrafos descritivos de diferentes estilos de vinculação adulta, o que permite a sua classificação do seu estilo de vinculação como seguro, inseguro-ansioso/ambivalente ou inseguro-evitante.

\section{Avaliação das relações profissionais}

A Medida Categorial de Avaliação da Orientação para o Trabalho, pretende avaliar a orientação face ao trabalho segundo a mesma lógica da medida categorial do estilo de vinculação adulta. $\mathrm{O}$ indivíduo tem de escolher entre três parágrafos descritivos dos seus sentimentos face ao trabalho, o que permite a classificação do seu estilo de orientação para o trabalho como segura, insegura-ansiosa/ambivalente ou insegura-evitante.

A Escala de Satisfação no Trabalho, de tipo Likert: é constituída por 11 itens $\left(\alpha=0,83^{4}\right)$, avalia o nível de satisfação dos indivíduos em relação

\footnotetext{
${ }^{4}$ Alphas de Cronbach na amostra desta investigação.
} 
à actividade profissional (i.e., segurança, variedade de tarefas, remuneração e desafio) e às relações no contexto de trabalho.

A Escala de Insatisfação no Trabalho, composta por 10 itens $(\alpha=0,85)$, de tipo Likert, avalia o nível de insatisfação dos indivíduos quanto a aspectos relacionados com o contexto laboral, nomeadamente segurança, variedade de tarefas, remuneração e desafio.

A Escala de Avaliação de Sentimentos face à Actividade Profissional, constituída por 27 itens $(\alpha=0,81)$, de tipo Likert, os quais pretendem avaliar a forma como o sujeito se relaciona com o trabalho. É composta pelas seguintes sub-escalas: Medo de Falhar (4 itens, $\alpha=0,60$ ), Prefere Trabalhar com Outros (4 itens, $\alpha=0,76), O$ Trabalho Prejudica as Relações e a Saúde (4 itens, $\alpha=0,63)$, Preocupação (6 itens, $\alpha=0,55)$, Não Reconhecido/Pouco apreciado (3 itens, $\alpha=0,56)$, Necessidade de se Sentir Ocupado (3 itens, $\alpha=0,46)$ e Motivado pela Aprovação (3 itens, $\alpha=0,35)$.

\section{Resultados}

\section{Vinculação}

Estilos de Vinculação

Tal como apresenta o Quadro 3, o estilo de vinculação seguro foi encontrado em $67,4 \%$ dos indivíduos. No que toca aos estilos de vinculação inseguros, $15,4 \%$ dos sujeitos foram avaliados como inseguros-evitantes e $17,2 \%$ como inseguros-ansiosos/ambivalentes, não tendo sido encontrada qualquer associação entre os estilos de vinculação e o género $\left(\chi^{2}(2)=\right.$ 1,13 , n.s.).

Quadro 3: Estilos de vinculação em função do género

\begin{tabular}{lccc}
\hline \multicolumn{2}{c}{ Género } \\
\hline Estilos de Vinculação & Masculino & Feminino & Total \\
\hline Seguro & $195(32,6 \%)$ & $208(34,8 \%)$ & $403(67,4 \%)$ \\
Inseguro-Ansioso/Ambivalente & $42(7,0 \%)$ & $50(8,4 \%)$ & $92(15,4 \%)$ \\
Inseguro-Evitante & $44(7,4 \%)$ & $59(9,9 \%)$ & $103(17,2 \%)$ \\
\hline
\end{tabular}




\section{Actividade Profissional}

Orientação para o Trabalho

De acordo com o Quadro 4, em relação à Orientação para o Trabalho, verifica-se que $66,5 \%(\mathrm{~N}=395)$ dos participantes se identifica com uma orientação segura, $25,6 \%(\mathrm{~N}=152)$ com uma orientação insegura-ansiosa/ambivalente e 7,9\% ( $\mathrm{N}=47)$ com uma orientação insegura-evitante. Também não foi encontrada uma associação significativa entre esta variável e o género $\left(\chi^{2}(2)=2,18\right.$, n.s.).

Quadro 4: Orientação para o trabalho em função do género

\begin{tabular}{lccc}
\hline \multicolumn{4}{c}{ Género } \\
\hline Orientação para o Trabalho & Masculino & Feminino & Total \\
\hline Segura & $183(30,8 \%)$ & $212(35,7 \%)$ & $395(66,5 \%)$ \\
Insegura-Ansiosa/Ambivalente & $70(11,8 \%)$ & $82(13,8 \%)$ & $152(25,6 \%)$ \\
Insegura-Evitante & $27(4,5 \%)$ & $20(3,4 \%)$ & $47(7,9 \%)$ \\
\hline
\end{tabular}

A satisfação profissional relaciona-se negativamente com as restantes medidas. Assim, valores elevados de satisfação face ao trabalho estão associados à percepção de que o trabalho realizado não é reconhecido pelos outros e a uma reduzida necessidade de se sentir ocupado. A motivação para a realização de tarefas no trabalho em indivíduos satisfeitos tende a ser pouco baseada na aprovação dos outros e o seu nível de preocupação é baixo. Por último, uma acentuada satisfação profissional está associada a uma baixa percepção de que o trabalho prejudica a saúde e as relações.

Valores elevados no medo de falhar na execução das tarefas laborais estão relacionados com uma elevada preferência em trabalhar com outros em detrimento de trabalhar sozinho e com valores elevados na preocupação face às tarefas laborais. Indivíduos com acentuado medo de falhar tendem a apresentar, ainda, valores elevados na percepção de que o trabalho individual não é reconhecido pelos pares no contexto profissional e, simultaneamente, consideram que a motivação face ao contexto laboral está ligada à aprovação dos pares e dos superiores hierárquicos. Ainda que refiram que a actividade profissional prejudica as relações e a saúde, apresentam valores elevados na necessidade de estar ocupado.

De salientar ainda que indivíduos com elevados níveis de motivação pela aprovação tendem a estar mais insatisfeitos no contexto laboral, a manifestar maior medo de falhar e maior preferência para trabalhar com os outros. Adicionalmente, revelam maiores índices de preocupação e necessidade de estar ocupado e relatam um menor reconhecimento pelos outros. 
Associação entre as medidas relacionadas com o Trabalho/Actividade Profissional

Uma vez que havia várias medidas para avaliação da actividade e relações profissionais, foram analisadas as correlações existentes, as quais são apresentadas no Quadro 5.

Quadro 5: Correlações entre as medidas de avaliação da actividade e relações profissionais

\begin{tabular}{|c|c|c|c|c|c|c|c|c|c|}
\hline & Sat & Insat & MF & PTO & TPSR & Preoc & NR & NEO & MA \\
\hline$\overline{\text { Satisfação (Sat) }}$ & - & & & & & & & & \\
\hline Insatisfação (Insat) & $-0,20 * * *$ & - & & & & & & & \\
\hline Medo de Falhar (MF) & 006 & 0,06 & - & & & & & & \\
\hline $\begin{array}{l}\text { Preferência por } \\
\text { Trabalhar com } \\
\text { Outros (PTO) }\end{array}$ & $-0,01$ & $-0,04$ & $0,14 * * *$ & - & & & & & \\
\hline $\begin{array}{l}\text { Trabalho Prejudica } \\
\text { Saúde e Relações } \\
\text { (TPSR) }\end{array}$ & $-0,12 * *$ & $0,18^{* * *}$ & $0,36^{* * *}$ & 0,01 & - & & & & \\
\hline Preocupação (Preoc) & $-0,09 *$ & $0,18^{* * *}$ & $0,38 * * *$ & $-0,02$ & $0,58 * * *$ & - & & & \\
\hline $\begin{array}{l}\text { Não Reconhecido } \\
\text { (NR) }\end{array}$ & $-0,16^{* * *}$ & $0,27^{* * *}$ & $0,37 * * *$ & $-0,04$ & $0,48 * * *$ & $0,46^{* * *}$ & - & & \\
\hline $\begin{array}{l}\text { Necessidade } \\
\text { de Estar Ocupado } \\
\text { (NEO) }\end{array}$ & $-0,09 *$ & $0,07^{+}$ & $0,27 * * *$ & $-0,04$ & $0,44 * * *$ & $0,39 * * *$ & $0,27 * * *$ & - & \\
\hline $\begin{array}{l}\text { Motivado } \\
\text { pela aprovação (MA) }\end{array}$ & $-0,11^{* *}$ & $0,10^{*}$ & $0,39 * * *$ & $0,14^{* * *}$ & $0,34 * * *$ & $0,46^{* * *}$ & $0,20 * * *$ & $0,32 * * *$ & - \\
\hline
\end{tabular}

Nota: $+\mathrm{p}<0,10 ; * \mathrm{p}<0,05 ; * * \mathrm{p}<0,01 ; * * \mathrm{p}<0,001$

A satisfação profissional relaciona-se negativamente com as restantes medidas. Assim, valores elevados de satisfação face ao trabalho estão associados à percepção de que o trabalho realizado não é reconhecido pelos outros e a uma reduzida necessidade de se sentir ocupado. A motivação para a realização de tarefas no trabalho em indivíduos satisfeitos tende a ser pouco baseada na aprovação dos outros e o seu nível de preocupação é baixo. Por último, uma acentuada satisfação profissional está associada a uma baixa percepção de que o trabalho prejudica a saúde e as relações.

Valores elevados no medo de falhar na execução das tarefas laborais estão relacionados com uma elevada preferência em trabalhar com outros em detrimento de trabalhar sozinho e com valores elevados na preocupação face às tarefas laborais. Indivíduos com acentuado medo de falhar tendem a apresentar, ainda, valores elevados na percepção de que o trabalho indivi- 
dual não é reconhecido pelos pares no contexto profissional e, simultaneamente, consideram que a motivação face ao contexto laboral está ligada à aprovação dos pares e dos superiores hierárquicos. Ainda que refiram que a actividade profissional prejudica as relações e a saúde apresentam valores elevados na necessidade de estar ocupado.

De salientar ainda que indivíduos com elevados níveis de motivação pela aprovação tendem a estar mais insatisfeitos no contexto laboral, a manifestar maior medo de falhar e maior preferência para trabalhar com os outros. Adicionalmente, revelam maiores índices de preocupação e necessidade de estar ocupado e relatam um menor reconhecimento pelos outros.

Quadro 6: Orientação para o trabalho e actividade e relações profissionais

\begin{tabular}{|c|c|c|c|c|c|c|c|}
\hline \multirow[b]{3}{*}{$\begin{array}{l}\text { Relações Profis- } \\
\text { sionais }\end{array}$} & \multicolumn{4}{|c|}{ Orientação para o trabalho } & \multicolumn{3}{|c|}{ Post-Hoc (Gabriel) } \\
\hline & \multirow[b]{2}{*}{$\begin{array}{l}\text { Segura } \\
\left(\begin{array}{c}\mathrm{N}=395) \\
\mathrm{M}(\mathrm{dp})\end{array}\right.\end{array}$} & \multicolumn{2}{|c|}{ Insegura } & \multirow[b]{2}{*}{$F(2,591)$} & \multirow[b]{2}{*}{ S vs. E } & \multirow[b]{2}{*}{ S vs. P } & \multirow[b]{2}{*}{ E vs. P } \\
\hline & & $\begin{array}{c}\text { Ansiosa/ } \\
\text { /Ambivalente } \\
(\mathrm{N}=152) \\
\mathrm{M}(\mathrm{dp})\end{array}$ & $\begin{array}{l}\text { Evitante } \\
(\mathrm{N}=47) \\
\mathrm{M}(\mathrm{dp})\end{array}$ & & & & \\
\hline Satisfação & $31,71(4,93)$ & $30,36(5,59)$ & $28,85(5,53)$ & $8,75^{* * *}$ & $* * *$ & * & n.s. \\
\hline Insatisfação & $22,41(5,87)$ & $23,77(5,42)$ & $24,72(5,71)$ & $5,47 * *$ & * & * & n.s. \\
\hline Medo de Falhar & $10,23(1,70)$ & $11,69(1,78)$ & $11,00(2,00)$ & $39,05 * * *$ & $* *$ & $* * *$ & $*$ \\
\hline $\begin{array}{l}\text { Preferência de } \\
\text { Trabalhar com } \\
\text { Outros }\end{array}$ & $10,23(2,00)$ & $11,72(1,93)$ & $8,25(1,77)$ & $63,21 * * *$ & $* * *$ & $* * *$ & $* * *$ \\
\hline $\begin{array}{l}\text { Trabalho Preju- } \\
\text { dica Saúde e } \\
\text { Relações }\end{array}$ & $8,30(1,72)$ & $9,51(2,06)$ & $9,95(2,07)$ & $34,39 * * *$ & $* * *$ & $* * *$ & n.s. \\
\hline Preocupação & $12,58(1,94)$ & $13,88(2,31)$ & $14,08(2,66)$ & $27,30 * * *$ & $* * *$ & $* * *$ & n.s. \\
\hline Não Reconhecido & $6,78(1,40)$ & $7,50(1,60)$ & $7,68(1,44)$ & $18,36 * * *$ & $* * *$ & $* * *$ & + \\
\hline $\begin{array}{l}\text { Necessidade de } \\
\text { Estar Ocupado }\end{array}$ & $5,66(1,46)$ & $6,22(1,57)$ & $6,74(1,85)$ & $15,47 * * *$ & $* * *$ & $* * *$ & + \\
\hline $\begin{array}{l}\text { Motivado pela } \\
\text { aprovação }\end{array}$ & $6,30(1,23)$ & $7,01(1,30)$ & $6,72(1,44)$ & $20,39 * * *$ & $*$ & $* * *$ & + \\
\hline
\end{tabular}

Nota: $+\mathrm{p}<0,10 ;{ }^{*} \mathrm{p}<0,05 ; * * \mathrm{p}<0,01 ; * * * \mathrm{p}<0,001$.

Orientação para o Trabalho e medidas relacionadas com a Actividade e Relações Profissionais

Como se pode observar no Quadro 6, foram encontradas diferenças significativas entre os participantes com diferentes orientações para o trabalho em termos das várias medidas relacionadas com a actividade profissional (Wilks' Lambda $=0,67, \mathrm{~F}(18,1166)=14,62, \mathrm{p}<0,001)$. 
Análises mais detalhadas, com recurso a testes univariados seguidos de testes post-hoc, revelaram que os indivíduos com uma orientação segura para o trabalho diferem significativamente de ambos os grupos inseguros, no sentido de revelarem maior satisfação e melhor adaptação ao trabalho nas diferentes medidas. Apenas ao nível da escala preferência por trabalhar com outros se verificou que o grupo seguro pontua entre os grupos inseguros. Finalmente, as únicas medidas em que os dois grupos inseguros evidenciaram diferenças foram no medo de falhar e preferência por trabalhar com outros: em ambas as escalas, os indivíduos com estilo ansioso/ambivalente apresentam médias mais elevadas do que aqueles que apresentam um estilo evitante.

\section{Estilos de Vinculação, Orientação para o Trabalho e Actividade e Relações Profissionais}

Associação entre Estilos de Vinculação e Orientação para o Trabalho

Foi encontrada uma relação significativa entre os Estilos de Vinculação e a Orientação para o Trabalho $\left(\chi^{2}(4)=51,25, \mathrm{p}<0,01\right)$, como ilustra o Quadro 7. Assim, a maioria dos indivíduos que se identifica com um estilo de vinculação seguro também se identifica com uma orientação segura para o trabalho $(\mathrm{N}=302,75,7 \%)$, os participantes com um estilo de vinculação ansiosa/ambivalente tendem a identificar-se com uma orientação para o trabalho também ansiosa/ambivalente $(\mathrm{N}=43,46,7 \%)$. Curiosamente, esta tendência não foi encontrada para os indivíduos com um estilo de vinculação evitante, os quais só minoritariamente se identificam com o mesmo tipo de orientação para o trabalho $(\mathrm{N}=13,12,6 \%)$.

\section{Estilos de Vinculação e Actividade e Relações Profissionais}

Há diferenças significativas entre os estilos de vinculação e diversas medidas de actividade e relações profissionais (Wilks' Lambda $=0,88$, $\mathrm{F}(18,1174)=4,44, p<0,001)$, como se pode verificar no Quadro 8. Testes univariados permitiram constatar a existência de diferenças significativas entre os três estilos de vinculação na maioria das dimensões consideradas. Indivíduos classificados com estilo de vinculação segura apresentam maiores níveis de satisfação relativamente ao contexto profissional do que os indivíduos com estilo de vinculação insegura, ansiosa/ambivalente ou evitante, os quais não diferem entre si. Uma vez mais, são os indivíduos seguros que revelam maior satisfação com o contexto profissional e menor medo de falhar; para além disso, são os que menos consideram que a actividade profissional prejudica as relações interpessoais e a saúde individual 
e que, no contexto de trabalho, consideram ter menos preocupação face à realização e conclusão das tarefas.

Quadro 7: Estilos de Vinculação em função da Orientação para o Trabalho

\begin{tabular}{lcccc}
\hline \multirow{2}{*}{ Estilos de Vinculação } & \multicolumn{2}{c}{ Orientação para o Trabalho } & \multirow{2}{*}{ Total } \\
\cline { 2 - 4 } & Segura & $\begin{array}{c}\text { Ansiosa/ } \\
\text { /Ambivalente }\end{array}$ & Evitante & \\
\hline \multirow{2}{*}{ SEGURO } & $302(50,8 \%)$ & $75(12,6 \%)$ & $22(3,7 \%)$ & $399(67,2 \%)$ \\
Ansioso/Ambivalente & $37(6,2 \%)$ & $43(7,2 \%)$ & $12(2,0 \%)$ & $92(15,5 \%)$ \\
Evitante & $56(9,4 \%)$ & $34(5,7 \%)$ & $13(2,2 \%)$ & $103(17,3 \%)$ \\
\hline Total & $395(66,5 \%)$ & $152(25,6 \%)$ & $47(7,9 \%)$ & 594 \\
\hline
\end{tabular}

Quadro 8: Estilos de Vinculação e Actividade e Relações Profissionais

\begin{tabular}{|c|c|c|c|c|c|c|c|}
\hline \multirow[b]{3}{*}{$\begin{array}{l}\text { Relações Profis- } \\
\text { sionais }\end{array}$} & \multicolumn{4}{|c|}{ Estilo de Vinculação } & \multicolumn{3}{|c|}{ Post-Hoc (Gabriel) } \\
\hline & \multirow[b]{2}{*}{$\begin{array}{l}\text { Seguro } \\
\mathrm{N}=403 \\
\mathrm{M}(\mathrm{dp})\end{array}$} & \multicolumn{2}{|c|}{ Inseguro } & \multirow[b]{2}{*}{$\mathrm{F}(2,595)$} & \multirow[b]{2}{*}{ S vs. E } & \multirow[b]{2}{*}{ S vs. P } & \multirow[b]{2}{*}{ E vs. P } \\
\hline & & $\begin{array}{c}\text { Ansioso/ } \\
\text { /Ambivalente } \\
\mathrm{N}=92 \\
\mathrm{M}(\mathrm{dp})\end{array}$ & $\begin{array}{l}\text { Evitante } \\
\mathrm{N}=103 \\
\mathrm{M}(\mathrm{dp})\end{array}$ & & & & \\
\hline Satisfação & $31,62(5,11)$ & $30,26(5,21)$ & $29,96(5,35)$ & $5,77 * *$ & $* *$ & $*$ & n.s. \\
\hline Insatisfação & $22,49(5,87)$ & $24,20(5,82)$ & $23,46(5,31)$ & $3,83 *$ & n.s. & $*$ & n.s. \\
\hline Medo de Falhar & $10,40(1,82)$ & $11,54(1,77)$ & $10,93(1,84)$ & $16,10 * * *$ & * & $* * *$ & + \\
\hline $\begin{array}{l}\text { Preferência de } \\
\text { Trabalhar com } \\
\text { Outros }\end{array}$ & $10,47(2,10)$ & $10,68(10,87)$ & $10,22(2,65)$ & 1,109 & - & - & - \\
\hline $\begin{array}{l}\text { Trabalho Preju- } \\
\text { dica Saúde e } \\
\text { Relações }\end{array}$ & $8,47(1,83)$ & $9,47(2,02)$ & $9,12(2,07)$ & $12,73 * * *$ & $* *$ & $* * *$ & n.s. \\
\hline Preocupação & $12,73(2,11)$ & $13,65(2,24)$ & $13,64(2,33)$ & $11,77 * * *$ & $* * *$ & $* * *$ & n.s. \\
\hline Não Reconhecido & $6,74(1,39)$ & $7,78(1,66)$ & $7,53(1,50)$ & $26,32 * * *$ & $* * *$ & $* * *$ & n.s. \\
\hline $\begin{array}{l}\text { Necessidade de } \\
\text { Estar Ocupado }\end{array}$ & $5,79(1,51)$ & $6,16(1,60)$ & $6,04(1,69)$ & $2,78+$ & n.s. & + & n.s. \\
\hline $\begin{array}{l}\text { Motivado pela } \\
\text { aprovação }\end{array}$ & $6,39(1,21)$ & $6,89(1,28)$ & $6,63(1,25)$ & $6,89 * * *$ & n.s. & $* * *$ & n.s. \\
\hline
\end{tabular}

Nota: $+\mathrm{p}<0,10 ; * \mathrm{p}<0,05 ; * * \mathrm{p}<0,01 ; * * * \mathrm{p}<0,001$. 


\section{Discussão e Conclusões}

Os resultados revelam que $67,4 \%$ dos sujeitos se identifica com o estilo de vinculação segura e $32,6 \%$ como insegura, sendo que, dentro destes, $17,2 \%$ se identificam como ansiosos/ambivalentes e $15,4 \%$ como evitantes. De um modo geral, estes resultados vão ao encontro daqueles encontrados nos estudos pioneiros de Hazan e Shaver (1987, 1990), Shaver e Hazan (1988), Shaver, Hazan e Bradshaw (1988) e de outros posteriores (Van IJzendoorn \& Bakermans-Krannenburg, 1996; Campos, Barrett, Lamb, Goldsmith, \& Stenberg, 1983; Feeney \& Noller, 1990; Levy \& Davis, 1988; Mikulincer, Florian \& Tolmacz, 1990). No entanto, na amostra portuguesa, a percentagem de indivíduos com estilo ansioso/ambivalente é superior à dos evitantes.

No que se refere à Orientação para o Trabalho, $66.5 \%$ dos participantes tem uma Orientação Segura, 25,6\% evidencia uma Orientação Ansiosa/Ambivalente e 7,9\% uma Orientação Evitante. Em contraste com os indivíduos com uma orientação insegura, os que evidenciam uma orientação segura para o trabalho demonstram uma maior satisfação e melhor adaptação ao trabalho nas diferentes medidas utilizadas. De sublinhar que os valores elevados de satisfação face ao trabalho estão associados a uma baixa percepção de que o trabalho realizado não é reconhecido pelos outros e uma reduzida necessidade de se sentirem ocupados. A motivação para a realização de tarefas no contexto de trabalho de indivíduos satisfeitos tende a ser pouco baseada na aprovação dos outros e o seu nível de preocupação é baixo, acontecendo o mesmo relativamente à percepção de que o trabalho prejudica a saúde e as relações.

O medo de falhar e a preferência por trabalhar com outros são as variáveis que marcam diferença entre os indivíduos com Orientação insegura para o Trabalho: os sujeitos classificados com uma orientação insegura-ansiosa/ambivalente têm mais medo de falhar e uma maior preferência de trabalhar com os outros do que os que são classificados como tendo uma orientação insegura-evitante para o trabalho, necessitam da presença/proximidade dos outros para que lhes seja possível explorar. Com medo de falhar e motivados pela aprovação de terceiros, a presença de colegas de trabalho parece marcar a cadência do seu trabalho, pela importância da sua aprovação e por diluir a responsabilidade individual no resultado final da actividade laboral.

Por outro lado, valores elevados no medo de falhar na execução das tarefas laborais têm inerente uma elevada percepção de que o trabalho individual não é reconhecido pelos pares, no contexto profissional, e, simultaneamente, a motivação face ao contexto laboral aparece ligada à aprovação dos pares e dos superiores hierárquicos. Ainda que considere que a 
actividade laboral prejudica as relações e a saúde pela exigência que implica e pela quantidade de horas que dedica ao contexto profissional, este grupo de indivíduos sente necessidade de estar ocupado em actividades desta natureza, tendo tendência a sobreocupar-se, quer pela necessidade de agradar aos outros assumindo múltiplas tarefas, quer pela exigência que colocam em cada tarefa que realiza.

No presente estudo, tal como em Hazan e Shaver (1990), foi encontrada uma relação significativa entre o Estilo de Vinculação e a Orientação para o Trabalho. Em termos análogos aos resultados de Ainsworth e colaboradores (1978), que apontam para diferenças no comportamento de exploração em crianças com diferentes padrões de vinculação, também o presente estudo permite identificar diferenças individuais no comportamento exploratório face ao mundo profissional, em estreita relação com os diferentes estilos de vinculação dos adultos. Em termos conceptuais, as representações internas que os indivíduos construíram a partir das suas histórias de vinculação poderão ajudar a compreender esta relação. Assim, um indivíduo que construiu modelos internos do self como competente e capaz de suscitar respostas positivas do outro, com valor e merecedor de afecto tenderá a percepcionar os outros como disponíveis, responsivos e dignos de confiança, a sentir-se também mais capaz e disponível para explorar mais e com maior confiança as oportunidades que o mundo do trabalho lhe proporciona.

Os indivíduos com estilos de vinculação segura e evitante apresentam na sua maioria $(75,7 \%$ e $54,4 \%$ respectivamente) uma Orientação $\mathrm{Se}$ gura no Trabalho, referem que os seus sentimentos e postura face ao trabalho se caracterizam por: a) ser confortável trabalhar sozinho ou com outras pessoas; b) retirar prazer do trabalho; c) não se preocupar com a possibilidade de falhar; d) não procrastinar; e) trabalhar arduamente; f) antecipar com entusiasmo e apreciar as férias; g) não permitir que o trabalho interfira com as relações e/ou com a saúde. A maioria dos indivíduos com estilo de vinculação inseguro-ansioso/ambivalente evidencia uma Orientação Insegura-Ansiosa/Ambivalente no Trabalho e percepciona o contexto de trabalho em termos de: a) preferir trabalhar com alguém do que sozinho; b) desejar que os outros reconheçam, elogiem e agradeçam o esforço realizado; c) preocupação com a possibilidade de falhar; d) preocupação que o fracasso implique a perda de afecto e respeito das outras pessoas.

Nesta linha, parece-nos que um aspecto que pode merecer uma maior atenção incide na relação entre o estilo de vinculação evitante e a orientação evitante para o trabalho. A maioria dos sujeitos com estilo de vinculação evitante apresenta, como referimos, uma orientação segura no trabalho, o que poderá causar estranheza. Estabelecendo um paralelismo entre o comportamento exploratório das crianças e dos adultos, poderíamos levantar a hipótese que os adultos evitantes terão uma orientação para o trabalho 
no sentido de uma maior exploração, mas de menor qualidade - o que os distinguiria dos indivíduos com estilo seguro. No entanto, o tipo de instrumento utilizado não nos permite analisar a qualidade desse exploração.

Tal como no estudo de Hazan e Shaver (1990), os sujeitos com estilo de vinculação seguro apresentam maiores níveis de satisfação relativamente ao contexto profissional do que os indivíduos com estilo de vinculação inseguro, ansioso/ambivalente ou evitante. Em termos das relações profissionais, os indivíduos com estilo seguro são os que consideram menos que não são reconhecidos ou que são pouco apreciados no contexto laboral, pelos colegas e superiores hierárquicos. Relativamente às tarefas laborais, tendem a manifestar menos preocupação face à realização e conclusão das tarefas e menos medo de falhar. Na relação da actividade profissional com a vida pessoal, são os que consideram menos que a actividade profissional prejudica as relações interpessoais e a saúde individual.

Centrados no comportamento exploratório, não estando sujeitos a pressões internas para fugir ou procurar mais intensamente a satisfação das necessidades de vinculação, os indivíduos com estilo de vinculação seguro tenderão a explorar activamente e com qualidade, conseguindo obter do contexto de trabalho recompensas inerentes à própria actividade desenvolvida. Como Cassidy (2001) sublinhou, os indivíduos classificados com um estilo de vinculação seguro têm expectativas de que os outros os aceitam, assim como às suas imperfeições, o que poderá contribuir muito para um bom relacionamento com os colegas de trabalho; ao conseguirem distanciar-se das necessidades de vinculação (porque satisfeitas), poderão explorar de forma autónoma os desafios que o contexto profissional lhes proporciona, sentindo-se gratificados com os sucessos e as competências adquiridas no percurso.

Os resultados obtidos estão em consonância com o estudo de Hazan e Shaver (1990), relativamente aos indivíduos com estilo de vinculação ansioso/ambivalente: são os que manifestam níveis mais elevados de insatisfação face à actividade profissional (aspecto em que se diferenciam estatisticamente do estilo seguro); nas relações profissionais tendem a considerar-se mais vezes como pouco apreciados e/ou não reconhecidos pelos colegas no contexto profissional, estando a motivação para a execução das tarefas subordinada à aprovação dos mesmos. Em relação à concretização e execução das tarefas, este grupo de indivíduos apresenta uma tendência para níveis mais elevados de medo de falhar e para considerar que a actividade profissional prejudica a qualidade das relações interpessoais e a saúde.

Neste sentido, os resultados parecem sugerir que o comportamento exploratório está ao serviço do sistema de vinculação, pelo investimento no trabalho. Os indivíduos com estilo ansioso/ambivalente parecem procurar satisfazer as suas necessidades de vinculação; uma vez que consideram que 
só pela realização profissional poderão aceder ao afecto dos outros e da figura de vinculação, per si, não são merecedores de amor e responsividade por parte da figura de vinculação.

Os indivíduos com estilo de vinculação evitante exprimem menor satisfação com o contexto de trabalho, mas não são os mais insatisfeitos. Como a investigação tem apontado (Shaver \& Mikulincer, 2002), o grupo evitante tende a comunicar apenas sentimentos positivos ou menos positivos, o que poderá emergir da estratégia de contenção no sentido de menor activação de emoções negativas.

Em síntese, os resultados mostram que diferentes estilos de vinculação estão associados a diferenças individuais na orientação face ao trabalho. Este estudo tem, contudo, limitações que não podem deixar de ser apontadas, nomeadamente, a medida do estilo de vinculação, que, sendo uma medida categorial e levando a uma escolha forçada, implica uma perda de informação qualitativa.

O contexto profissional assume-se como de primordial importância na idade adulta, constituindo uma tarefa desenvolvimental organizada e organizadora do próprio desenvolvimento do indivíduo Parece-nos claro que o aprofundamento do conhecimento desta problemática exige procedimentos de avaliação mais complexos e compreensivos, nomeadamente tendo por base a avaliação dos padrões de representação da vinculação (e não apenas dos estilos de vinculação) e da sua relação com os comportamentos, sentimentos e atitudes face ao contexto de trabalho. Nesta linha e a título de exemplo, destacaríamos a entrevista utilizada por Sroufe, Egeland, Carlson e Collins (2005), no estudo longitudinal de Minnesota, designada de "23 years school/work experience interview".

$\mathrm{Na}$ sequência deste estudo sobre Vinculação e Trabalho, está em curso uma investigação com base em medidas mais robustas, em termos conceptuais e psicométricos, no sentido de contribuir para o conhecimento mais vasto sobre a vinculação num

"movimento em espiral que retoma e reconstrói, a níveis diferentes de complexidade e de profundidade, questões colocadas previamente e, simultaneamente, integra novos elementos, permitindo a transformação e gerando a inovação" (Soares, 2002, p. 1).

\section{Referências}

Ainsworth, M. (1989). Attachment Beyond Infancy. American Psychologist, 44, 4. 709-716.

Ainsworth, M., \& Bell, S. (1970). Attachment, Exploration and Separation: Illustrate by the Behaviour of one-year-olds in a Strange Situation. Child Development, 41, 49-67. 
Ainsworth, M., Blehar, M., Waters, E., \& Wall, S. (1978). Patterns of attachment: A Psychological Study of the Strange Situation. Hillsdale: Erlbaum.

Bartholomew, K., \& Horowitz, L. (1991). Attachment styles among young adults: A test of a four-category model. Journal of Personality and Social Psychology, 61, 2, 226-244.

Bowlby, J. (1969). Attachment and loss: Attachment. London: Basic Books (edição revista, 1982).

Bowlby, J. (1973). Attachment and loss: Separation. London: Basic Books.

Bowlby, J. (1979). The making and breaking of affectional bonds. London: Tavistock.

Bowlby, J. (1980). Attachment and loss: Loss, sadness and depression. London: Basic Books.

Campos, J. J., Barrett, K. C., Lamb, M. E., Goldsmith, H. H., \& Stenberg, C. (1983). Socioemotional Development. In M. M. Haith \& J. J. Campos (Eds.), Handbook of Child Psychology. Vol. 2: Infancy and Psychobiology (pp. 783-915). New York: Wiley.

Carlson, E. \& Sroufe, A. (1995). Contribution of attachment theory to developmental psychopathology. In D. Cicchetti \& D. Cohen (Eds.), Development and Psychopathology. Vol. 1: Theory and Method (pp. 581-617). NY: Wiley.

Cassidy, J., (2001). True, lies and intimacy: An attachment perspective. Attachment and Human Development, 3, 2, 121-155.

Cassidy, J., \& Shaver, P. (Eds.) (1999). Handbook of attachment: Theory, research and clinical applications. NY: The Guilford Press.

Cicchetti, D., \& Cohen, D. (1995a). Development and Psychopathology. Vol. 1: Theory and Methods. NY: Wiley.

Cicchetti, D., \& Cohen, D. (1995b). Development and Psychopathology. Vol. 2: Risk, Disorder and Adaptation. NY: Wiley.

Collins, N., Guichard, A., Ford, M., \& Feeney, B. (2005). Working Models of Attachment New developments and emerging themes. In Rholes \& Simpson (Eds.), Adult Attachment Theory, Research, and Clinical Implications. London: The Guiford Press.

Cowan, P., Cohn, D., Cowan, C., \& Pearson, J. (1996). Parents' attachment histories and children's externalizing and internalizing behavior: Exploring family systems models of linkage. Journal of Consulting and Clinical Psychology, 64, 53-63.

Feeney, J. A., \& Noller, P. (1990) Attachment style and verbal descriptions of romantic partners. Journal of Social and Personal Relationship, 8, 187-215.

Feeney, J. A., \& Noller, P. (1996) Adult Attachment. Sage Publications, Inc.

Feeney, J. A., Noller, P., \& Patty, J. (1993). Adolescents' interactions with opposite sex: influence of attachment style and gender. Journal of Adolescent, 16, 169$-186$.

Grossmann, K.E. (1999). Old and new internal working models of attachment: The organization of feelings and language. Attachment \& Human Development, 1, 3, 253-269. 
Hazan, C., \& Shaver, P. (1987). Romantic Love Conceptualized as an Attachment Process. Journal of Personality and Social Psychology, 52, 3, 511, 524.

Hazan, C., \& Shaver, P. (1990). Love and Work: An attachment Theoretical perspective. Journal of Personality and Social Psychology, 59, 270-280.

Hazan, C., \& Shaver, P. (1994). Attachment as an organizational framework for research on close relationship. Psychological Inquiry, 5, 1-22.

Hazan, C., \& Zeifman, D. (1994). Sex and Psychological tether. In K. Bartholomew \& D. Perlman (Eds.), Advances in personal relationships (vol. 5, 151-178). London: Jessica Kingsley.

Hazan, C. \& Zeifman, D. (1999). Pair bonds as attachment: Evaluating the evidence. In J. Cassidy \& P. Shaver (Eds.), Handbook of attachment: Theory, research and clinical applications (pp. 355-377). NY: The Guilford Press.

Jongenelen, I. (2004). Mães Adolescentes e Bebés: Da Matriz Relacional à Matriz Contextual. Dissertação apresentada para o grau de Doutor em Psicologia na Universidade do Minho. Manuscrito não Publicado.

Kobak, R., \& Sceery A. (1988). Attachment in late adolescence: working models, affect regulation, and representations of self and others. Child Development, 59, 135-146.

Kunce, L., \& Shaver, P. (1984). An Attachment - Theoretical Approach to Caregiving in Romantic Relationships. In K. Bartholomew \& D. Perlman (Eds.), Advances in personal relationships (vol. 5, 205-237). London: Jessica Kingsley.

Levy, M., \& Davis, K. (1988). Love styles and attachment styles compared: Their relations to each other and to various relationship characteristics. Journal of Social and Personal Relationships, 5, 439-471.

Main, M., Kaplan, N., \& Cassidy, J. (1985). Security in infancy, childhood, and adulthood: A move to the level of representation. In I. Bretherton \& E. Waters (Eds.), Growing points of attachment theory and research. Monographs of the Society for Research in Child Development, 50 (1-2, serial n. ${ }^{\circ}$ 209), 66-104.

Matos, P. (2002). (Des)continuidades na Vinculação aos Pais e ao Par Amoroso em Adolescentes. Dissertação apresentada para o grau de Doutor em Psicologia na Universidade do Porto, Faculdade de Psicologia e Ciências da Educação.

Mikulincer, M., Florian, V., \& Tolmacz, R. (1990). Attachment styles and fear of personal death: A case study of affect regulation. Journal of Personality and Social Psychology, 58, 273-280.

Pianta, R., Marvin, R., \& Morog, M. (1999). Resolving the past and present: Relations with attachment organization. In J. Solomon \& C. George (Eds.), Attachment Disorganization (pp. 379-398). NY: The Guilford Press.

Rholes, S., Simpson, J. (2004). Adult Attachment: theory, research, and clinical implications. The Guilford Press. NY.

Shaver, P., \& Hazan, C. (1988). A based overview of the study of love. Journal of Social and Personal Relationships, 5, 473-501.

Shaver, P., Hazan, C., \& Bradshaw, D. (1988). Love as attachment: The integration of three behavioural systems. In R. Sternberg \& M. Barnes (Eds.), The Psychology of Love (pp. 69-99). New Haven, CT: Yale University Press. 
Shaver, P., \& Mikulincer, M. (2002). Dialogue on adult Attachment: Diversity and integration. Attachment and Human Development, 4, 2, 243-257.

Simpson, J. (1990). Influence of attachment styles on romantic relationships. Journal of Personality and Social Psychology, 59 (5), 971-980.

Soares, I. (1996). Representação da vinculação na idade adulta e na adolescência. Estudo intergeracional: mãe-filho(a). Braga: Centro de Estudos em Educação e Psicologia, Instituto de Educação e Psicologia, Universidade do Minho (2. ${ }^{\text {a }}$ edição em 2000).

Soares, I. (2000). Introdução à Psicopatologia do Desenvolvimento: Questões teóricas e de investigação. In I. Soares (Ed.), Psicopatologia do Desenvolvimento: Trajectórias (in)adaptativas ao longo da vida (pp. 13-42). Coimbra: Quarteto.

Soares, I. (2000). Psicopatologia do Desenvolvimento e contexto familiar: Teoria e investigação das relações de vinculação. In I. Soares (Ed.), Psicopatologia do Desenvolvimento: Trajectórias (in)adaptativas ao longo da vida (pp. 381-434). Coimbra: Quarteto.

Soares, I. (2001). Vinculação e cuidados maternos: Segurança, protecção e desenvolvimento da regulação emocional no contexto da relação mãe-bebé. In C. Canavarro (Ed.), Psicologia da Gravidez e da Maternidade (pp. 75-104). Coimbra: Quarteto.

Soares, I. (2002). A Vinculação Vinculada. Lição de síntese apresentada às provas de agregação (não publicada). Departamento de Psicologia. Universidade do Minho.

Soares, I., Fremmer-Bombik, E., Grossmann, K. E., \& Silva, C. (2000). Attachment representation in adolescence and adulthood: an intergenerational and cultural approach. In P. Crittenden (Ed.), The Organization of Attachment Relationship: Maturation, Culture, and Context (pp. 325-342). Cambridge: Cambridge University Press.

Solomon, J., \& George, C. (1996). Defining the caregiving system: Toward a theory of caregiving. Infant Mental Health Journal, 17, 3, 183-197.

Sroufe, A., Carlson, E., \& Shulman, S. (1993). Individuals in relationships: Development from infancy through adolescence. In D. Funder, R. Parke, C. Tomlinson-Keasey \& K. Widaman (Eds.), Studying lives through time: Personality and development (pp. 315-342). Washington, DC: APA.

Sroufe, A., Cooper, R., \& DeHart, G. (1996). Child development. Its nature and course. NY: McGraw hill.

Sroufe, A., Egeland, B., Carlson, E., \& Collins, A. (2005). The Development of the person:tthe Minnesota study of risk and adaptation from birth to adulthood. The Guilford Press. NY.

Sroufe, A., \& Waters, E. (1977). Attachment as an organizational construct. Child Development, 48, 1184-1199.

Van IJzendoorn, M., \& Bakermans-Krannenburg, M. (1996). Attachment representations in mothers, fathers, adolescents, and clinical groups: A meta-analytic search for normative data. Journal of Consulting and Clinical Psychology, 64, $8-21$. 
Van IJzendoorn, M., Goldberg, S., Kroonenberg, P., \& Frenkel, O. (1992). The relative effects of maternal and child problems on the quality of attachment: A meta-analysis of attachment in clinical samples. Child Development, 63, 840-858 .

Waters, E., \& Cummings, M. (2000). A secure base from which to explore close relationships. Child Development, 71, 1, 164-1272.

Waters, E., Merrick, S., Treboux, D., Crowell, J., \& Albersheim, L. (2000). Attachment security in infancy and early adulthood: A twenty-year longitudinal study. Child Development, 71, 3, 684-689.

Waters, E., Weinfield, N., \& Hamilton, C. (2000). The stability of attachment security from infancy to adolescence and early adulthood: General discussion. Child Development, 71, 3, 703-706. 